\title{
High-throughput novel microsatellite marker of faba bean via next generation sequencing
}

Tao Yang ${ }^{1 \dagger}$, Shi-ying Bao ${ }^{2 \dagger}$, Rebecca Ford ${ }^{3}$, Teng-jiao Jia', Jian-ping Guan ${ }^{1}$, Yu-hua He${ }^{2}$, Xue-lian Sun ${ }^{1}$, Jun-ye Jiang ${ }^{1}$, Jun-jie Hao ${ }^{4}$, Xiao-yan Zhang ${ }^{4}$ and Xu-xiao Zong ${ }^{1 *}$

\begin{abstract}
Background: Faba bean (Vicia faba L.) is an important food legume crop, grown for human consumption globally including in China, Turkey, Egypt and Ethiopia. Although genetic gain has been made through conventional selection and breeding efforts, this could be substantially improved through the application of molecular methods. For this, a set of reliable molecular markers representative of the entire genome is required.

Results: A library with 125,559 putative SSR sequences was constructed and characterized for repeat type and length from a mixed genome of 247 spring and winter sown faba bean genotypes using 454 sequencing. A suit of 28,503 primer pair sequences were designed and 150 were randomly selected for validation. Of these, 94 produced reproducible amplicons that were polymorphic among 32 faba bean genotypes selected from diverse geographical locations. The number of alleles per locus ranged from 2 to 8 , the expected heterozygocities ranged from 0.0000 to 1.0000 , and the observed heterozygosities ranged from 0.0908 to 0.8410 . The validation by UPGMA cluster analysis of 32 genotypes based on Nei's genetic distance, showed high quality and effectiveness of those novel SSR markers developed via next generation sequencing technology.
\end{abstract}

Conclusions: Large scale SSR marker development was successfully achieved using next generation sequencing of the $V$. faba genome. These novel markers are valuable for constructing genetic linkage maps, future QTL mapping, and marker-assisted trait selection in faba bean breeding efforts.

Keywords: Microsatellite markers, Next generation sequencing, Marker development, Vicia faba L.

\section{Background}

Faba bean (Vicia faba L.) is an important temperate legume, grown for human consumption and animal feed due to its high protein and fibre content $[1,2]$. The crop also replaces available nitrogen in the soil when used in rotation with cereals and oilseeds, and thus is expected to be a highly beneficial component in future temperate Low Carbon Agricultural systems. China is the largest faba bean producer $(40.36 \%)$ with an average dry grain production (2005-2009) of 1,720,000 metric tonnes (mt) from 945,400 hectares; followed by Ethiopia (476,026 mt), France $(331,122 \mathrm{mt})$, Egypt $(274,040 \mathrm{mt})$ and Australia $(196,800 \mathrm{mt})$ [3].

\footnotetext{
*Correspondence: zongxx@mail.caas.net.cn

${ }^{\dagger}$ Equal contributors

'Institute of Crop Science, The National Key Facility for Crop Gene Resources and Genetic Improvement, Chinese Academy of Agricultural Sciences, Beijing 100081, China

Full list of author information is available at the end of the article
}

However, faba bean suffers from several major biotic and abiotic factors that constrain productivity. Although significant genetic gain to overcome these has been made through traditional breeding practices [1], progress through the use of genomics and associated biotechnologies is limited. This is due mainly to the large genome size (13GB; [4]), which is approximately 25 times larger than that of the model legume Medicago truncatula, and 2.5 times larger than Pisum sativum [1], together with a lack of financial investment in this crop species.

Recent advances in next generation sequencing (NGS) technologies enable the generation of large volumes of sequence efficiently and cost-effectively $[5,6]$. This has led to a revolution in biological and agricultural applications including identification of genes correlated with key breeding traits through high-density SNP marker and genome-wide association analysis studies (GWAS) $[7,8]$. Another outcome is the ability to accurately identify sequences flanking simple sequence repeat (SSR) regions

\section{Biomed Central}


Table 1 Occurrence of microsatellites in the genome survey

\begin{tabular}{ll}
\hline Category & Numbers \\
\hline Total number of sequences examined & 532,599 \\
\hline Total size of examined sequences (bp) & $162,448,842$ \\
\hline Total number of identified SSRs & 250,393 \\
\hline Number of SSR containing sequences & 125,559 \\
\hline Number of sequences containing more than one SSR & 61,266 \\
\hline Number of SSRs present in compound formation & 122,988 \\
\hline
\end{tabular}

for use as locus-specific markers for downstream genotyping. Otherwise known as microsatellites, SSRs are tandemly repeated motifs of 1 to 6 nucleotides found in both coding and non-coding regions $[9,10]$. These have become a marker of choice in many genotyping applications due to their relatively high abundance, high level of allelic variation, co-dominant inheritance, analytical simplicity and transferability of results across laboratories [11].

A limited number of characterized SSR loci $(<120)$ which have been validated over relatively few genetic backgrounds are available for faba bean. Initially, Pozarkova et al. developed primers to 25 SSR loci detected in chromosome 1 DNA libraries [12]. Subsequently, Zeid et al. developed primers to 54 SSR loci [13] and Gong et al. developed 11 EST-SSR loci primers [14]. Most recently, EST sequences within the public domain databases were screened and an additional 21 novel SSR loci were characterized and validated among 32 faba bean accessions [15].
Besides providing a cost-effective valuable source for molecular marker generation, the identification of SSR within ESTs is an effective approach for gene discovery and transcript pattern characterization, particularly if through mapping an EST-SSR or EST marker is significantly associated with a QTL [16-18]. This may be achieved by searching for SSR associated sequences within EST of a well characterised crop or model plant species. Together with the advantage of in silico analysis, this approach has the potential to substantially broaden the field of comparative studies to species where limited or no sequence information is available.

The present study identified high-quality putative SSR loci and flanking primer sequences cheaply and efficiently using the Roche 454 GS FLX Titanium platform. The resultant SSR sequences were characterized and validated through successful amplification of randomly selected target loci across a selection of faba bean genotypes from diverse geographic origin.

\section{Methods}

\section{Plant material}

A total of 247 faba bean accessions were selected from the National Genebank of China held at the Institute of Crop Science (ICS), Chinese Academy of Agricultural Sciences (CAAS), Beijing. Of these, 100 originated from China, 54 were from other Asian countries, 39 were from Europe, 30 were from Africa, 14 were from the America, 9 breeding lines were sourced from the

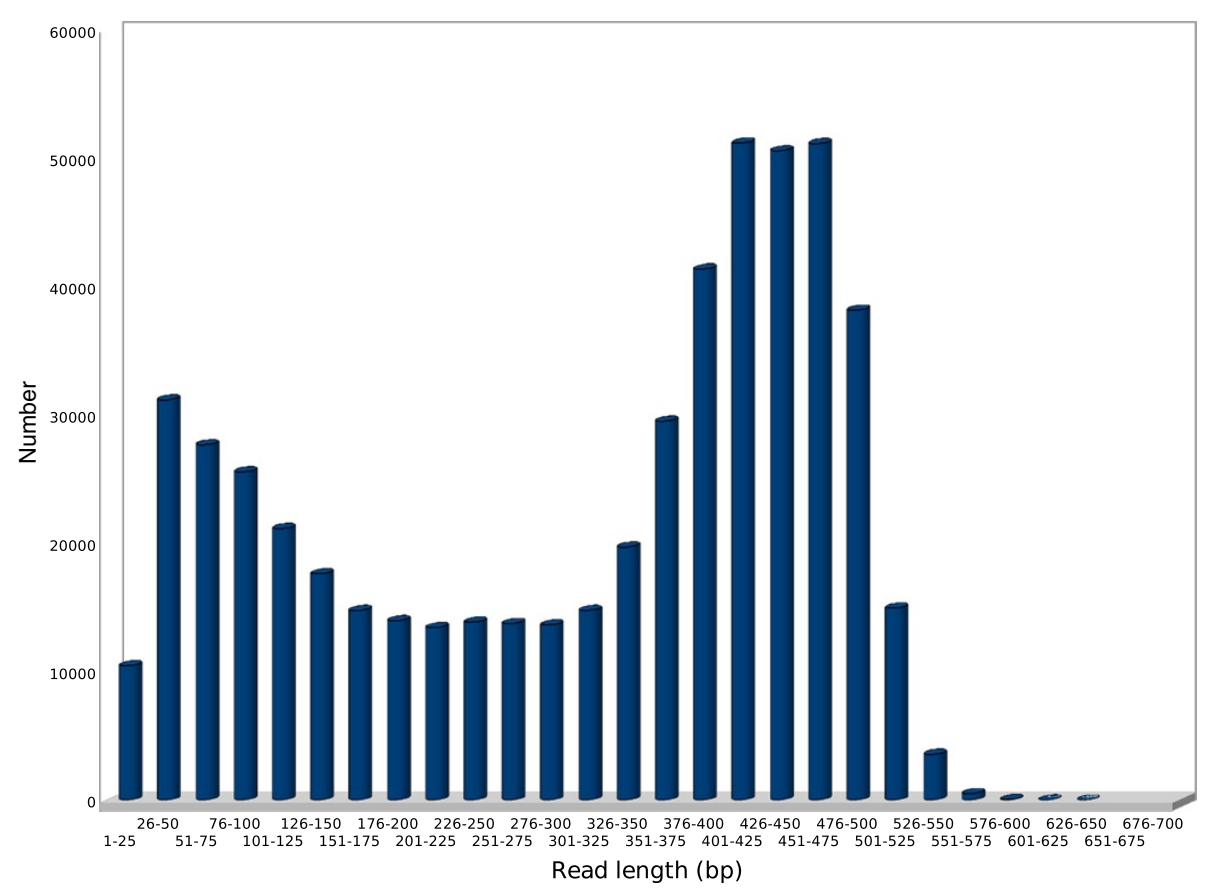

Figure 1 Frequencies length distribution of 454 read sequences. 


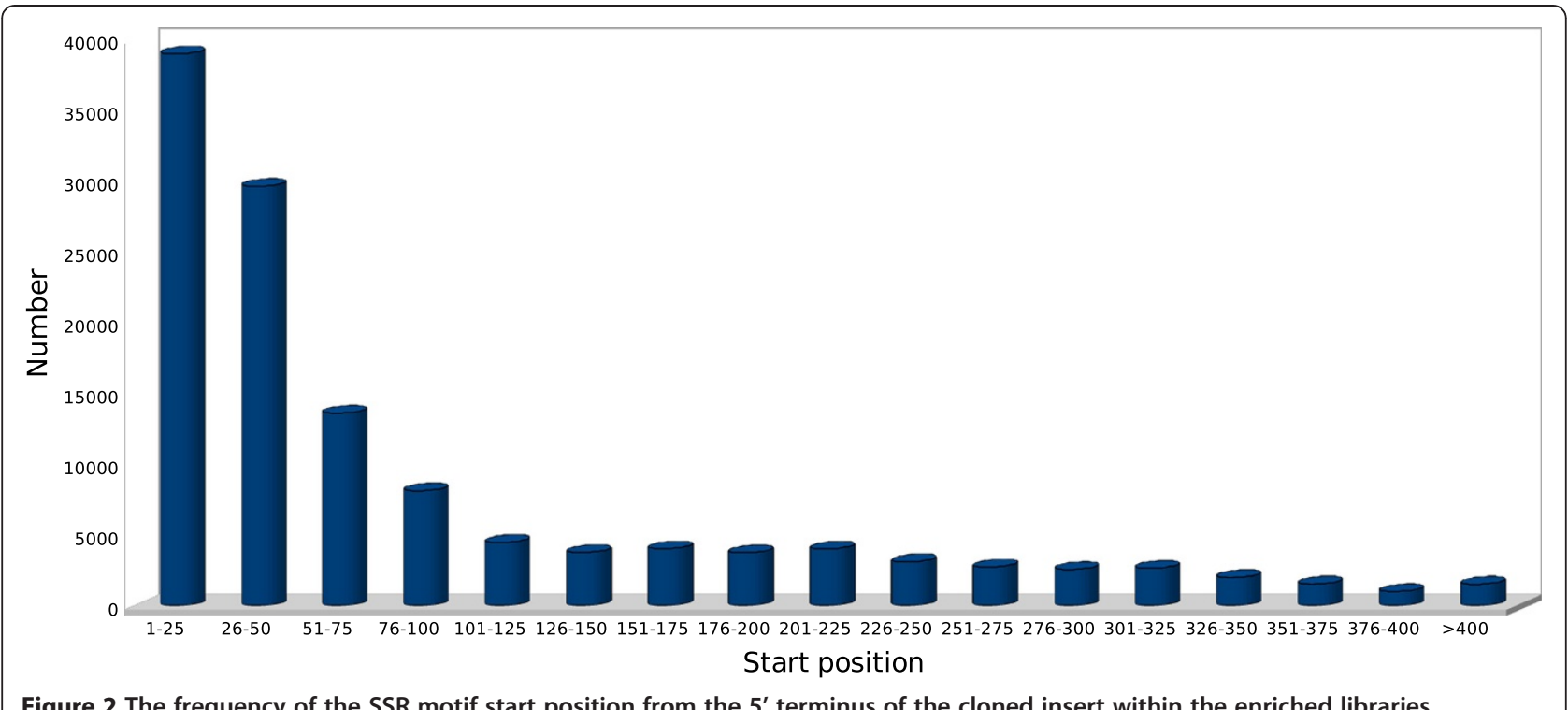

ICARDA (International Center for Agricultural Research in the Dry Areas) faba bean breeding program and one was from Oceania (Additional file 1: Table S1).

\section{DNA isolation, library preparation and 454 sequencing}

Seven days after seed were left on moist filter paper in the dark at $22^{\circ} \mathrm{C}$, sprouts from each of the 247 genotypes were collected. A single sprout of each genotype and of approximately the same weight was pooled and total gDNA was extracted using the CTAB method $[19,20]$.

Genome libraries were constructed using eight biotin labeled probes and a selective hybridization with streptavidin coated bead method [21-23]. The probes were: pGA, pAC, pAAT, pAAC, pAAG, pATGT, pGATA and
pAAAT. The quality of libraries was inspected by randomly selecting and sequencing 276 clones. The cloning vector was pEASY-T1 (TransGen Biotechnology Co., Ltd), and the primers used for sequencing were F: $5^{\prime}-\mathrm{G}$ TAAAACGACGGCCAGT-3' and R: 5'-CAGGAAAC AGCTATGAC-3'. Libraries were considered to be of high quality if the length of sequences were from 200 to $1000 \mathrm{bp}$, as evidenced on agarose gel.

Subsequently, entire libraries were equally pooled and subjected to 454 sequencing with GS-FLX Titanium reagents at Beijing Autolab Biotechnology Co., Ltd (China). All processing and analyses of the sequencing data was performed with GS-FLX Software v2.0.01 (454 Life Sciences, Roche, Germany). Using a series of normalization,

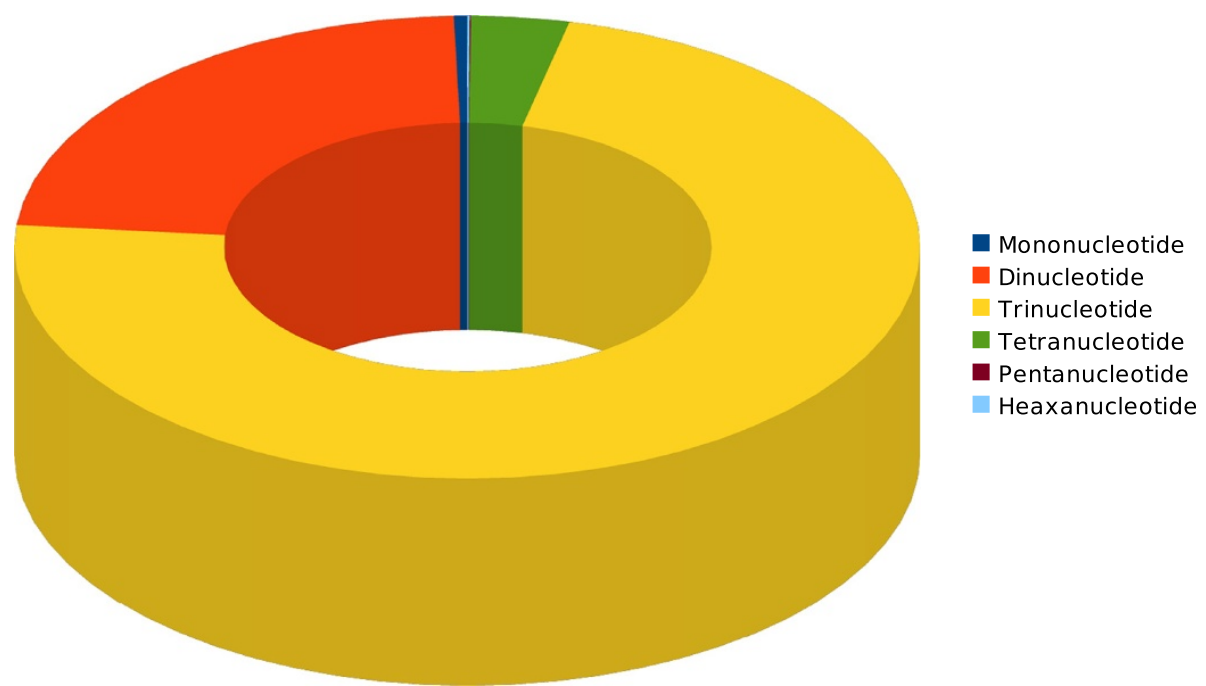

Figure 3 Frequencies of different nucleotide repeat sizes within the clones analysed. 
Table 2 Characteristics of 94 polymorphic SSR markers developed in Vicia faba $\mathrm{L}$. (F=forward primer, R=reverse primer, Size $=$ size of cloned allele, $\mathrm{Ta}=$ annealing temperature)

\begin{tabular}{|c|c|c|c|c|c|}
\hline Primer & Repeat & $F\left(5^{\prime}-3^{\prime}\right)$ & $R\left(5^{\prime}-3^{\prime}\right)$ & $\begin{array}{l}\text { Size } \\
\text { (bp) }\end{array}$ & $\begin{array}{l}T a \\
\left({ }^{\circ} \mathrm{C}\right) \\
\end{array}$ \\
\hline CAAS1 & $(\mathrm{AAAGGG}) 7$ & AGTCAGGGGGTCGATTTTTC & TCTTGCGCAGTTTTGACATC & 212 & 55 \\
\hline CAAS2 & $(\mathrm{GAA}) 9$ & TACAAAAGCTCTGGGGCCTA & CCAATTCCTCTGGGCAACT & 202 & 56 \\
\hline CAAS3 & $(A G) 7$ & CTGGTGCGTAAGGTTGATGA & CAAACCACCACCAATCACAG & 132 & 53 \\
\hline CAAS4 & (CA)11 & ATTGCAAGTCCTGAGGCAAG & ATAATGGCGCCACAAAGTGT & 160 & 57 \\
\hline CAAS5 & $(A C A) 15$ & TACATCAGTCCCGCAAATCA & CCATGTAGCCGATTCCACTT & 150 & 55 \\
\hline CAAS6 & (A) 10 & TGCAAAGTAATTCCGAAACAA & CGCACATGAATTGGGGTAAT & 150 & 56 \\
\hline CAAS7 & (A) 10 & GACCCAAGCCTTCACCACTA & TGTGTGGGATCCATITTGAA & 200 & 59 \\
\hline CAAS8 & $(\mathrm{AAC}) 14$ & AATTTGTTCAGCATCTCGGG & CTGGTTGGTTCCTGGTGAGT & 150 & 56 \\
\hline CAAS9 & $(\mathrm{AAC}) 9$ & GTGATGCTITGCCTGTGCTA & ATGGACGTTTGTAGGTGGGA & 200 & 56 \\
\hline CAAS10 & $(\mathrm{AAG}) 5$ & CTGTTCGTCATCATCATCGG & CGTAAATCAACCCCAACACC & 150 & 53 \\
\hline CAAS11 & $(A C A) 10$ & TCCCGCTATTCTTGCTCTGT & GCTCAAAAATGCTTGTCTTTCA & 170 & 54 \\
\hline CAAS12 & (TGT)9 & GAGGAGGATCCCACAATGAA & GCCAAAAGAGCCATGGTAGA & 210 & 56 \\
\hline CAAS13 & $\begin{array}{l}\text { (CAA) } \\
11 \text { aaatcccaaaaactgcaaattgtatgccatcttaaaccatac } \\
\text { (CAA)7 }\end{array}$ & CAAAAATCCCAAAAACTGCAA & TCGATITITCGACTTGGGTC & 130 & 56 \\
\hline CAAS14 & $(\mathrm{AAC}) 6$ & CCGTAGATCTCAAAAACCATGA & GGAGGAAGGAAGCTCGAATC & 170 & 60 \\
\hline CAAS15 & $(A A C) 8$ & AACCAACATCAATGGCATCA & TCTITCCTITTCCTCTTCCA & 140 & 60 \\
\hline CAAS16 & $(C A) 7$ & TCAAATTTCCCTTTGCAAAAAT & GACCAAGGTCAACCACCTTI & 350 & 56 \\
\hline CAAS17 & $(C A) 8$ & TCAAACACCTACACACCCACA & TCTCGGTCAATCTCACATGC & 250 & 56 \\
\hline CAAS18 & $(C A) 9$ & ATGGGAGGGCAAATTTTAGG & AGTGAGTGGAGCGCTTGTTI & 350 & 56 \\
\hline CAAS19 & $(\mathrm{CAA}) 6$ & AACATITITCCAATCGAGGC & TGTAGGCTTACGGCCAAAGA & 200 & 56 \\
\hline CAAS20 & (CAT)5 & ACTGGAAAATCCCAATGCAC & AGCAAACTTGCACCCAACAT & 190 & 56 \\
\hline CAAS21 & $(\mathrm{CTT}) 8$ & GAATTITCAAAACATGAGTCCCA & CCGGATCTGAAAAGACTTGC & 175 & 60 \\
\hline CAAS22 & $(\mathrm{G}) 10$ & TGATGAACAGAACTGCGCTC & ATTGGAGAGAGGCGAAATCA & 190 & 56 \\
\hline CAAS23 & $(\mathrm{GA}) 6$ & ACCGCATGCTAGGGAGTCTA & TGGGTGACTCACTITTGTGG & 220 & 58 \\
\hline CAAS24 & (GA)6gca(AG)6(TG)8 & TCACTCACAAGCCACTAAGTCAA & GATGCGACACTATCCCCACT & 200 & 56 \\
\hline CAAS25 & (GT)15 & TCCATAATCAATTGGCTAAGCTC & AAGACTAACTCTCGACTGTATTTAGGC & 150 & 58 \\
\hline CAAS26 & $(\mathrm{GT}) 7$ & CGGCTTGGTTAACTGGATGT & TCTTCCTITTCTTCAATGCG & 160 & 58 \\
\hline CAAS27 & $(\mathrm{TA}) 6$ & TTGGCATCATGCTCTAATCG & CTTGAAGTCGTGCCAGATGA & 280 & 60 \\
\hline CAAS28 & (TC)8 & CCATTGATGCAGGAAAGGAT & CAGCTTTGACAGCTCCAACA & 160 & 58 \\
\hline CAAS29 & $(\mathrm{TCA}) 5$ & TGCAAGTCAGTAGCCAAGACA & СTCGTCTCTCCTCATTCCCA & 180 & 58 \\
\hline CAAS30 & (TG) 10 & GGTTTTAGGTGATTTTCGCA & GCGAAACCTCGTATGGTTGT & 170 & 59 \\
\hline CAAS31 & (TG) 12 & CAACGCGCTAGAGGAAGAAG & CCACTGCCCTAGCACACTAA & 160 & 56 \\
\hline CAAS32 & (TG)7 & TाTGGGGTACAACACTGGGT & ССТСАСТССТСТАТАTAAACAACACTT & 200 & 59 \\
\hline CAAS33 & $(\mathrm{TGA}) 5$ & GCAGTGATTCTGGCAGTGAA & TGCAGCAACATTTCCATCAT & 190 & 56 \\
\hline CAAS34 & (TGT)5 & TTTCTCGCAATTGTTCTCACA & TTCGATGAAATCCATCTTCTGA & 200 & 57 \\
\hline CAAS35 & (TTG)8 & AGGCAGAAGTTGGAAGCAA & TCTCACTTCGGCTTCAGGAT & 180 & 56 \\
\hline CAAS36 & (A) 11 & AGCACTAGAGTTCCAAGCCA & TIITATCGTITCTTGTCACGC & 130 & 52 \\
\hline CAAS37 & (A) 11 & CAACGCAAGAACACGTGAAT & TAGAGGCCAATTCAAGCCAT & 190 & 54 \\
\hline CAAS38 & $(\mathrm{AAC}) 5$ & CGCCTCAGAACCAAGTTCAT & TGCTITGTTITGGTTTTGTGA & 170 & 56 \\
\hline CAAS39 & $(\mathrm{AAG}) 5$ & CTGTTCGTCATCATCATCGG & CGTAAATCAACCCCAACACC & 170 & 54 \\
\hline CAAS40 & $(\mathrm{AAG}) 6$ & CCAAAGCCACTTCCAAACAT & TTCAGCCGGGCTTCTTTC & 110 & 54 \\
\hline CAAS41 & $(\mathrm{AC}) 10$ & GAAACCCACTTGGTCGTGTC & TTCATTTGGGTAGGCTCCAA & 190 & 56 \\
\hline CAAS42 & $(\mathrm{AC}) 10$ & CAAGTGTCGACGCAAGAGAT & TGACTTITTGACTGCTCCCA & 250 & 56 \\
\hline CAAS43 & $(A C) 7$ & GAGGAAGTGTGAAAGGTCGC & TCATTITAAAGTGGTGTATGTGTGT & 170 & 54 \\
\hline
\end{tabular}


Table 2 Characteristics of 94 polymorphic SSR markers developed in Vicia faba $\mathrm{L}$. (F=forward primer, R=reverse primer, Size $=$ size of cloned allele, $\mathrm{Ta}=$ annealing temperature) (Continued)

\begin{tabular}{|c|c|c|c|c|c|}
\hline CAAS44 & $(\mathrm{AC}) 7$ & ACACACACACGCACACACAC & CATGAACCTITGATAGTTTTCCA & 150 & 56 \\
\hline CAAS45 & $(A G A) 5$ & ATGGCTTTGACAAAAGGGAA & СTCCTTCACCCGACAATGTT & 180 & 57 \\
\hline CAAS46 & $(A G A) 6$ & AGATCGCAGGCGTAGAAAGA & TGCTTCAACCACAACACCAT & 200 & 58 \\
\hline CAAS47 & (C)11 & CAAATTGGTTTGCATATCCG & AGCCCTTCACATCCATTGAG & 200 & 56 \\
\hline CAAS48 & (CA)10 & ССTCCTCCTITAATTTGTGGC & TGAATCGTGAATGCTCTCTGA & 200 & 56 \\
\hline CAAS49 & (CA)10 & ACCTCCATAGCAGCAGCATT & GGCCAATTCTTAACGTGCTT & 140 & 56 \\
\hline CAAS50 & (CA)10 & CACTGGACCATTTTGCATTC & ATGAGATCCGGAGCAGATGT & 140 & 56 \\
\hline CAAS51 & (CA)11 & AAGCATTAAAACTCCCATAGCG & ATGTGTGCGTGTGTCATGTG & 140 & 52 \\
\hline CAAS52 & (CA)12 & CATTCCATGTTGCGTITITG & GGATAAGAGGGTGGTGGTGA & 200 & 56 \\
\hline CAAS53 & (CA)13 & GGCCCATTTGTAAGGGTTT & AATGAGATCTGGCCTGGATG & 200 & 56 \\
\hline CAAS54 & $(C A) 6$ & CCATTGGACCTCTTTGCATT & CCAGAGTGGATGATGATCTGA & 150 & 54 \\
\hline CAAS55 & (CA)6 & ACTCACATACACGCACACACA & AATGCTCTCATCCCTITTGC & 150 & 56 \\
\hline CAAS56 & (CA)6 & CACATACACGCACACACACA & AATGCTCTCATCCCTTITGC & 150 & 56 \\
\hline CAAS57 & $(C A) 8$ & GCCCGAGACACTTTGGTTTA & CCAGAATGGATGAGGACCTG & 210 & 56 \\
\hline CAAS58 & $(\mathrm{CA}) 9$ & CTCCTGGTCCATGTATGAATGA & TGTGTGTATGTGTATGCGTGC & 150 & 54 \\
\hline CAAS59 & (CAA) 10 & GGCCAACATAGGTGAGCATT & GTGTTGTAGGCCTTTGGTCC & 200 & 56 \\
\hline CAAS60 & $(C A A) 8$ & ATGCAAAATGAAATGCGACA & TGTAGTTGTCTGTTAATGGTTGTTG & 190 & 56 \\
\hline CAAS61 & (G)11 & AGAGGAAAAAGGCAAATGGC & CCCTTCATCAATCACACCAA & 130 & 54 \\
\hline CAAS62 & $(G A) 14$ & AATGTTGGGACGGAGTTCAG & TTGTTGATTCATTCATCCCTTG & 130 & 56 \\
\hline CAAS63 & (GA)15 & CGCAGAGAAACACTCCATGA & GAAGTTGAATGTCATTTGTGTCAA & 100 & 56 \\
\hline CAAS64 & $(G A) 6$ & AAAATATAATAAACAAAGCAAAAGTGC & CAGGTTTGTGGTTTCACCCT & 200 & 54 \\
\hline CAAS65 & $(\mathrm{GA}) 6$ & CGATATTCCTCGGTTTCCAA & CATGGGTCGTCTTCTCCACT & 200 & 54 \\
\hline CAAS66 & $(\mathrm{GA}) 6$ & CATCACTITCCAGCCTGTCA & ATTTCTGCCTCCCCTTGT & 190 & 58 \\
\hline CAAS67 & $(\mathrm{GA}) 7$ & GGGTTTCAGAGAAAGGGGTC & CGCAAGCGTATTGGGTATT & 130 & 56 \\
\hline CAAS68 & $(G A) 8$ & ATGGAGGTTGCGATTTGAAG & CATCATCTCCACACTIITTCCA & 130 & 54 \\
\hline CAAS69 & (GT)10 & ATTACAAATGTCGGTGCCGT & AGCACAACGATAAGATGATATGC & 170 & 54 \\
\hline CAAS70 & $(G T) 8$ & TCGCGATAGAGGTITTGGAA & AACAACAACGATTCATCACAAGA & 200 & 56 \\
\hline CAAS71 & (GTT)15 & CCATGTAGCCGATTCCACTT & TTCGGCAACGTAGGAAAAAT & 160 & 54 \\
\hline CAAS72 & (T)10 & TITTCCAGTGTCAACCCATCT & ACATGAGGCCAAAAACTGCT & 170 & 54 \\
\hline CAAS73 & (TG)13 & TTGCACCTCTGTTGAAGACG & TCACCAACACTCTAATCCTCAATC & 190 & 54 \\
\hline CAAS74 & $(\mathrm{AC}) 6$ & CCCACCGTATTACACAAGGG & GCGAGGAAGAAGATGACGTT & 200 & 56 \\
\hline CAAS75 & $(\mathrm{AG}) 15$ & TCGATTGCACAATAAATGGTTT & GAGGTCGACTCCCATTGAAA & 180 & 54 \\
\hline CAAS76 & $(\mathrm{AG}) 6$ & GCCTGTTAATGAGAAGAACTGGA & TITCAAAATTTAGTTTCTCTCTGTCTC & 200 & 56 \\
\hline CAAS77 & (CA)21 & TAGCAGCCAACAATCAGTGG & GGTGATGTTGCTCATGTTCG & 180 & 56 \\
\hline CAAS78 & (CA)7 & TCAAATTTCCCTTTGCAAAAAT & TCGAACACAACTTCTTCATTTCTC & 180 & 56 \\
\hline CAAS79 & (CA)7 & TCAAATTTCCCTTTGCAAAAAT & CATGGAAAATCTITATITTGTGTG & 100 & 58 \\
\hline CAAS80 & (CA)8 & GTGTGAAAACTCACCCGGTC & TGTGTGTAAGTGTGTGTATGTGTGTG & 130 & 54 \\
\hline CAAS81 & (GA)15 & AACTTACAGGGGCCACACAC & TGTGCATTATACTITACGTATGTTCCT & 100 & 52 \\
\hline CAAS82 & (GA)17 & TTGCTTGACAATGGTGGAA & ATTCAACAAGCAAGGGTTGG & 120 & 52 \\
\hline CAAS83 & (T)10 & GATTTGCGTTAAGGGTTCCA & GAACAAACTACGTTITATTGTCCAGA & 180 & 52 \\
\hline CAAS84 & $(\mathrm{TA}) 6$ & TGTCGACACCACAGCTATTIT & TGTGGTTCGTTGTTTTGGTG & 200 & 56 \\
\hline CAAS85 & (TCA)6 & TTGAAGTGAATAAGATGAAGAAGTGT & GTTGCCTTTCCTTGCATGAT & 130 & 56 \\
\hline CAAS86 & (TG)10 & TCGCGATAGAGGTITTGGAA & CACAAACAACAACGATTCATCA & 200 & 56 \\
\hline CAAS87 & (TG) 14 & CTCTACCATGGGCCATTTCT & AGAGATAGAGAGAGAGACAGAGATGAA & 90 & 54 \\
\hline CAAS88 & (TG)18 & TCCTACCGATCTCTCTCTCCC & GTGGCATAACCGCGTAAGTT & 130 & 56 \\
\hline$\overline{\text { CAAS89 }}$ & (TG)18 & TGTCTCGCCTTCAATCTTCC & CTTGCTAAGTGAGACTGCTGCT & 190 & 54 \\
\hline
\end{tabular}


Table 2 Characteristics of 94 polymorphic SSR markers developed in Vicia faba $L$. ( $F=$ forward primer, $R=$ reverse primer, Size $=$ size of cloned allele, $\mathbf{T a}=$ annealing temperature) (Continued)

\begin{tabular}{|c|c|c|c|c|c|}
\hline CAAS90 & (TG)19 & TCCATAGTCGATGAGGACCG & TTGTCTCATTGTCTTTCTTTTCTTTC & 100 & 54 \\
\hline CAAS91 & (TG)6 & ATCTTCGGCTTGGTTGATTG & GAGGCGGCCACATTAGACT & 200 & 56 \\
\hline CAAS92 & (TG)9 & CGAGATCTGGAGTGGATTTAGA & TITTCATATGCCACATGCTCA & 170 & 56 \\
\hline CAAS93 & $(\mathrm{TTC}) 5$ & GGCATTGCTTACTTACCGGA & CGACGTCGACATTAACATGC & 200 & 56 \\
\hline$\overline{\text { CAAS94 }}$ & (TTG)9 & TCCTCAACACGTGATGCAAT & TGTAGGACCAGGAAGGTCGT & 180 & 56 \\
\hline
\end{tabular}

correction and quality-filtering algorithms, the 454 sequencing data were processed to screen and filter for weak signals and low-quality reads, and to trim the read ends for 454 adaptor sequences using the EMBOSS [24] software package. The sequencing data were then submitted to the 'National Center for Biotechnology Information (NCBI) short read archive and given the accession number SRP006387.

\section{SSR loci search and primer design}

The software MISA (Microsatellite identification) tool (http://pgrc.ipk-gatersleben.de/misa/) was configured to locate a minimum of $10 \mathrm{bp}$ : monomers $(\times 10), 2$-mers $(\times 6), 3$-mers $(\times 5)$, 4-mers $(\times 5), 5$-mers $(\times 5)$ and 6 -mers $(\times 5)$. This tool allowed the identification and localization of perfect microsatellites as well as compound microsatellites. The maximum size of interruption allowed between two different SSR in a compound sequence was 100 bp. Subsequently, Primer 3.0 (http://www-genome. wi.mit.edu/genome_software/other/primer3.html.) was used to design primer pairs to the flanking sequences of each unique SSR.

\section{SSR characterization and validation}

The number of different types of SSR, length (motif bp $\times$ number of motifs) and SSR position was searched and analyzed for using a bespoke program written in MISA files [25] and plotted by OpenOffice.org Calc.

\section{Marker assessment}

Polymerase chain reactions (PCR) were performed in $20 \mu \mathrm{l}$ reaction volumes containing $0.5 \mathrm{U}$ of Taq DNA polymerase (Zhexing, Beijing, China), $1 \times$ PCR BufferII, $1.5 \mathrm{mM} \mathrm{MgCl}$, $25 \mu \mathrm{M}$ of dNTP, $0.4 \mu \mathrm{M}$ primer, and $50 \mathrm{ng}$ of genomic DNA. Microsatellite loci were amplified on a Heijingang Thermal Cycler (Eastwin, Beijing, China) with the following cycle: $5 \mathrm{~min}$ initial denaturation at $95^{\circ} \mathrm{C} ; 35$ cycles of $30 \mathrm{~s}$ at $95^{\circ} \mathrm{C}$, 30s at the optimized annealing temperature (Table 1), $45 \mathrm{~s}$ of elongation at $72^{\circ} \mathrm{C}$, and a final extension at $72^{\circ} \mathrm{C}$ for $10 \mathrm{~min}$. PCR products were initially assessed for size polymorphism on $6 \%$ denaturing polyacrylamide gels and visualized by silver nitrate staining.
The genotyping data was subsequently used to determine genetic relationships among $32 \mathrm{~V}$. faba accessions (eleven from China, seven from Asia, five from Europe, five from Africa, three from the Americas and one from Oceania; (Additional file 1: Table S1). The number of alleles (Na), expected $(\mathrm{He})$ heterozygosities and observed $(\mathrm{Ho})$ heterozygosities were calculated using POPGEN1.32 [26]. The cluster analysis of 32 genotypes was carried out based on Nei's unbiased measures of genetic distance [27] by using the unweighted pair-group method with arithmetic average (UPGMA), and the dendrogram was drawn by MEGA4 [28].

\section{Results}

\section{Quality inspection of the DNA library}

The recombination rate within the constructed SSRenriched V. faba library was $73.9 \%$. Among the 276 clones sequenced, $31.9 \%$ contained SSR sequences within an insert that ranged from 0.2 to $1.0 \mathrm{~kb}$ in size.

\section{4 sequencing and characterization reads}

A total of 578,251 reads were generated from the pooled library, and 532,599 read sequences were used for further analysis after adaptor removal. Adenine was the most abundant nucleotide (30\%), followed by thymine (27\%), guanine (22\%) and cytosine (21\%). The mean GC content was $43 \%$. The average length of read sequence was $305 \mathrm{bp}$, with a maximum length of 635 bp (Figure 1).

\section{Identification of SSR loci}

After MISA analysis, the number of sequences containing an SSR was 125,559, and in total 250,393 SSR loci were detected. The number of sequences containing more than one SSR loci was 61,266 and the number of SSRs present in compound formation was 122,988 (Table 1).

The total size of SSR motif sequences was 8,759,185 bp, with an average motif length of $69 \mathrm{bp}$. Of these, 25\% comprised more than one discrete repeat and a high proportion (49\%) was located within compound repeats. The majority of identified SSR motifs (83\%) were located between the 5'terminus and mid regions of the cloned sequences, and 
Table 3 Informativeness of SSR loci following amplification from 32 geographically diverse accessions of Vicia faba L

\begin{tabular}{|c|c|c|c|}
\hline \multirow[t]{2}{*}{ Locus } & \multicolumn{3}{|c|}{32 Accessions } \\
\hline & $\mathrm{Na}$ & $\mathrm{He}$ & Ho \\
\hline CAAS1 & 3 & 0.0000 & 0.3591 \\
\hline CAAS2 & 3 & 0.2857 & 0.5703 \\
\hline CAAS3 & 7 & 0.4444 & 0.8099 \\
\hline CAAS4 & 4 & 0.0000 & 0.6111 \\
\hline CAAS5 & 3 & 0.1111 & 0.6471 \\
\hline CAAS6 & 4 & 0.2188 & 0.6324 \\
\hline CAAS7 & 6 & 0.6774 & 0.7372 \\
\hline CAAS8 & 7 & 0.6250 & 0.8016 \\
\hline CAAS9 & 4 & 0.1290 & 0.7250 \\
\hline CAAS10 & 4 & 0.7419 & 0.7277 \\
\hline CAAS11 & 4 & 0.3929 & 0.6890 \\
\hline CAAS12 & 4 & 0.1000 & 0.6718 \\
\hline CAAS13 & 5 & 0.3871 & 0.6256 \\
\hline CAAS14 & 3 & 0.4062 & 0.6493 \\
\hline$\overline{\text { CAAS15 }}$ & 4 & 0.6129 & 0.6901 \\
\hline CAAS16 & 6 & 0.6667 & 0.7708 \\
\hline CAAS17 & 3 & 0.0000 & 0.5159 \\
\hline CAAS18 & 4 & 0.3333 & 0.6887 \\
\hline CAAS19 & 5 & 0.0500 & 0.7474 \\
\hline CAAS20 & 4 & 0.2593 & 0.5926 \\
\hline CAAS21 & 4 & 0.1562 & 0.4712 \\
\hline CAAS22 & 3 & 0.2222 & 0.6038 \\
\hline CAAS23 & 2 & 0.0938 & 0.0908 \\
\hline CAAS24 & 6 & 0.1000 & 0.8000 \\
\hline CAAS25 & 5 & 0.4375 & 0.7399 \\
\hline CAAS26 & 3 & 0.0000 & 0.6333 \\
\hline CAAS27 & 5 & 0.2963 & 0.7701 \\
\hline CAAS28 & 4 & 0.5294 & 0.6471 \\
\hline CAAS29 & 4 & 0.3793 & 0.4483 \\
\hline CAAS30 & 4 & 0.2917 & 0.4991 \\
\hline CAAS31 & 4 & 0.4167 & 0.3608 \\
\hline CAAS32 & 5 & 0.6875 & 0.7882 \\
\hline CAAS33 & 3 & 0.2188 & 0.6195 \\
\hline CAAS34 & 3 & 0.4091 & 0.5613 \\
\hline CAAS35 & 4 & 0.3226 & 0.6753 \\
\hline CAAS36 & 3 & 0.3182 & 0.6131 \\
\hline CAAS37 & 2 & 0.1053 & 0.1024 \\
\hline CAAS38 & 2 & 0.4500 & 0.5013 \\
\hline CAAS39 & 4 & 0.3226 & 0.5960 \\
\hline CAAS40 & 3 & 0.0000 & 0.3579 \\
\hline CAAS41 & 3 & 0.0645 & 0.5812 \\
\hline CAAS42 & 5 & 0.7500 & 0.7599 \\
\hline CAAS43 & 3 & 0.0000 & 0.6400 \\
\hline CAAS44 & 4 & 0.3333 & 0.6078 \\
\hline
\end{tabular}

Table 3 Informativeness of SSR loci following amplification from 32 geographically diverse accessions of Vicia faba L (Continued)

\begin{tabular}{|c|c|c|c|}
\hline CAAS45 & 4 & 0.1034 & 0.6068 \\
\hline CAAS46 & 3 & 0.0625 & 0.2758 \\
\hline CAAS47 & 5 & 0.0000 & 0.6885 \\
\hline CAAS48 & 3 & 0.5333 & 0.6706 \\
\hline CAAS49 & 3 & 0.0938 & 0.6424 \\
\hline CAAS50 & 4 & 0.2759 & 0.6733 \\
\hline CAAS51 & 4 & 1.0000 & 0.7270 \\
\hline CAAS52 & 3 & 0.7000 & 0.5757 \\
\hline CAAS53 & 5 & 0.5806 & 0.7832 \\
\hline CAAS54 & 5 & 0.6129 & 0.7441 \\
\hline CAAS55 & 3 & 0.0000 & 0.4504 \\
\hline CAAS56 & 2 & 0.5000 & 0.4944 \\
\hline CAAS57 & 5 & 0.2188 & 0.5045 \\
\hline CAAS58 & 3 & 0.4167 & 0.5616 \\
\hline CAAS59 & 5 & 0.5200 & 0.6686 \\
\hline CAAS60 & 3 & 0.8182 & 0.6104 \\
\hline CAAS61 & 3 & 0.2667 & 0.4881 \\
\hline CAAS62 & 2 & 0.6250 & 0.4583 \\
\hline CAAS63 & 3 & 0.1176 & 0.5704 \\
\hline CAAS64 & 4 & 0.4194 & 0.7229 \\
\hline$\overline{C A A S 65}$ & 4 & 0.4643 & 0.7266 \\
\hline CAAS66 & 4 & 0.3871 & 0.7123 \\
\hline$\overline{\text { CAAS67 }}$ & 4 & 0.0000 & 0.4719 \\
\hline CAAS68 & 2 & 0.2500 & 0.2283 \\
\hline CAAS69 & 6 & 0.9524 & 0.8072 \\
\hline CAAS70 & 2 & 0.0000 & 0.5034 \\
\hline CAAS71 & 6 & 0.1429 & 0.8097 \\
\hline CAAS72 & 2 & 0.1000 & 0.4808 \\
\hline CAAS73 & 5 & 0.2000 & 0.6220 \\
\hline CAAS74 & 3 & 0.1250 & 0.2651 \\
\hline CAAS75 & 5 & 0.2222 & 0.6797 \\
\hline CAAS76 & 4 & 0.1724 & 0.3358 \\
\hline CAAS77 & 5 & 0.3600 & 0.6106 \\
\hline CAAS78 & 5 & 0.6000 & 0.7734 \\
\hline CAAS79 & 5 & 0.2812 & 0.7941 \\
\hline CAAS80 & 4 & 0.6400 & 0.7192 \\
\hline CAAS81 & 5 & 0.0500 & 0.7167 \\
\hline CAAS82 & 4 & 0.6875 & 0.6230 \\
\hline CAAS83 & 4 & 0.6000 & 0.7590 \\
\hline CAAS84 & 3 & 0.0625 & 0.4172 \\
\hline CAAS85 & 3 & 0.3750 & 0.5928 \\
\hline CAAS86 & 3 & 0.0323 & 0.4691 \\
\hline CAAS87 & 5 & 0.9091 & 0.8139 \\
\hline CAAS88 & 6 & 0.8571 & 0.8269 \\
\hline CAAS89 & 8 & 0.0000 & 0.8410 \\
\hline CAAS90 & 4 & 0.5294 & 0.6471 \\
\hline
\end{tabular}


Table 3 Informativeness of SSR loci following amplification from 32 geographically diverse accessions of Vicia faba L (Continued)

\begin{tabular}{llll}
\hline CAAS91 & 5 & 0.8710 & 0.6267 \\
\hline CAAS92 & 4 & 0.3750 & 0.5382 \\
\hline CAAS93 & 4 & 0.1562 & 0.7217 \\
\hline CAAS94 & 5 & 0.2400 & 0.7412
\end{tabular}

Notes: Number of alleles $(\mathrm{Na})$, expected heterozygosity $(\mathrm{He})$ and observed heterozygosity $(\mathrm{Ho})$.

within $200 \mathrm{bp}$ of the 5'-terminus (Figure 2). A total of 28,503 primer pairs were designed for future assessment of locus amplification (Additional file 2: Table S2).

Abundance and length frequencies of SSR repeat motifs The most common SSR motifs comprised trinucleotide and dinucleotide repeats (Figure 3). The majority of the trinucleotide repeats were from 15 to $30 \mathrm{bp}$ in length. Within the 1,188 characterised mononucleotide SSR, $(\mathrm{A} / \mathrm{T})_{\mathrm{n}}$ was almost three times more common than $(\mathrm{C} / \mathrm{G})_{\mathrm{n}}$, particularly at the 11-12 bp length. The dinucleotide repeats $(\mathrm{AC} / \mathrm{GT})_{\mathrm{n}}$ and $(\mathrm{AG} / \mathrm{CT})_{\mathrm{n}}$ were predominant, representing $99.2 \%$ of all of the dinucleotides characterised. Triucleotide (AAC/GTT) $)_{n}$ repeats were the most abundant (96.5\%). Twenty two unique tetranucleotide repeat motifs were identified, with the most common being AGAT/ATCT (66.4\%), ACAG/ CTGT (19.3\%) and ACAT/ATGT (9.1\%). Pentanucleotide and hexanucleotide motifs were far less frequent, together comprising only $0.1 \%$ of the total SSR detected. The dominant pentanucelotide motif was AGAGT/ATCTC (23.8\%) and the most common hexanucelotide motif was ACACGC/CGTGTG (49.5\%) (Additional files 3, 4, 5, 6, 7 and 8: Figure S1-S6).

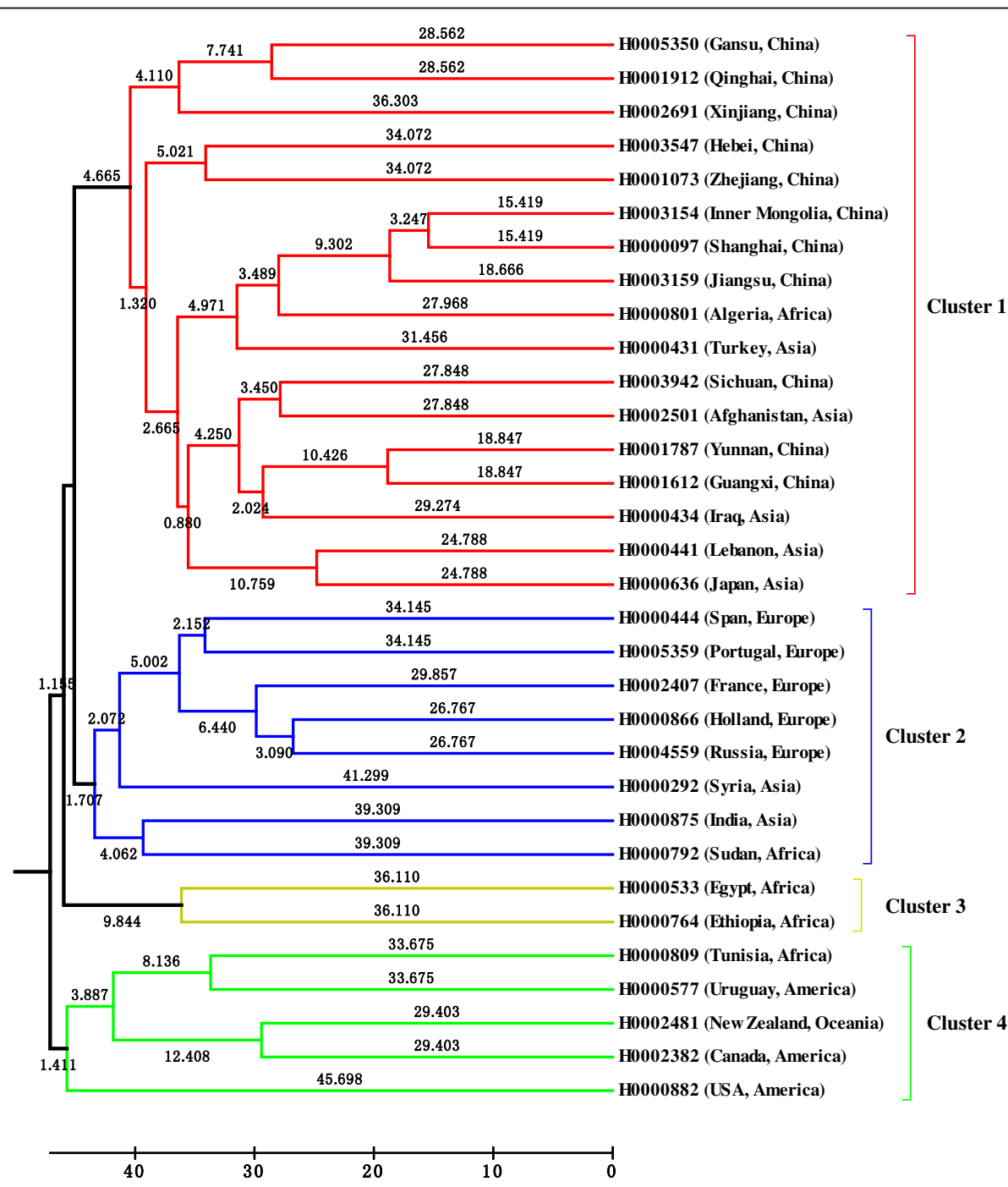

Figure 4 UPGMA dendrogram of 32 genotypes of faba bean. 


\section{Compound SSR analysis}

Two types of compound SSR were identified; those without an interruption between two motifs (ie (CA)12(ACG) 37 and noted as $C^{*}$ type) and those with an interruption between two motifs (ie (AAC)7gtcaat(AAC) 5 and noted as $C$ type). In total, $1,893 \mathrm{C}^{*}$ type and 59,369 $\mathrm{C}$ type compound SSR loci were detected among those sequenced, reflecting the complexity of the faba bean genome.

\section{Validation of SSR assay}

Of the 150 primer pairs selected for validation of SSR locus amplification, 102 produced a reproducible and clear amplicon of the expected size. Of these, 94 (63\%) were polymorphic among thirty-two genotypes assessed (Table 2). The number of alleles per locus ranged from 2 to 8 , the expected heterozygosities ranged from 0.0000 to 1.0000 , and the observed heterozygosities ranged from 0.0908 to 0.8410 (Table 3).

The dendrogram showed that the 32 faba bean genotypes fell into four distinct clusters (Figure 4). Cluster 1 comprised accessions from China and other Asian countries except for one accessions from Africa. Cluster 2 comprised accessions from Europe and nearby regions such as Syria. Cluster 3 comprised accessions from Africa and Cluster 4 contained accessions from America, Oceania and Africa. The pattern of diversity was similar to that previously observed using AFLP [29] and ISSR [30] markers.

\section{Discussion}

This study demonstrated that massively parallel sequencing technology offers opportunity to quickly identify large numbers of high quality SSR with diverse motifs from a genetically orphaned species such as Vicia faba. Given the huge number of marker loci identified in this study, future SSR marker optimisation may be best focussed on those comprising trinucleotide repeats. These repeats are generally more robust since they are reported to give fewer "stutter bands" than those based on dinucleotide repeats [31,32]. Also, trinucleotide repeats in particular have been demonstrated to be highly polymorphic and stably inherited in the human genome [33-35]. While the tri- and dinucleotide repeats mostly contributed to the major proportion of SSRs, a very small share was contributed by mono-, tetra-, penta- and hexa-nucleotide repeats. A similar trend was observed in other species [36].

The conversion of SSR-containing sequences into single locus markers may have a low success rate due to complex and/or insufficient flanking sequence. For example, just $20 \%$ of the identified dinucleotide repeats from spruce were converted to clear, discrete markers [37]. Similar observations were made for pine [38], wheat [39] and previously for $V$. faba [12]. Another factor affecting the development of clear markers is the complexity of the repeat motifs, indeed a high proportion of the SSR in the current study comprised compound repeats (49.1\%). Nevertheless, this study has provided the selected data required to potentially develop tens of thousands of novel SSR markers for the faba bean genome.

Previously, a total of 304,680 reads were generated and 802 EST-SSR primer pairs were designed from transcriptome sequencing of faba bean [40]. From this, 81 primer pairs were developed, of which $48 \%$ produced polymorphic markers on the genotypes assessed. In our study, 68\% (102) of the SSR loci identified were accurately amplified, of which 63\% (94) were polymorphic among the genotypes tested. This may be indicatative of the larger number of SSR loci detected, inclusive of nontranscribed sequences. Hence these markers may be more representative of the entire genome for the purposes of germplasm diversity assessment and conservation purposes [41]. Meanwhile, the identification of EST-SSR within sequences provides future opportunity to mine the expressed sequences for significant physical and functional association with traits of interest in markerassisted faba bean breeding.

\section{Conclusion}

This work represents a major advance in the identification of large numbers of informative SSR loci in $V$. faba by application of 454 GS FLX Titanium sequencing technology.

\section{Additional files}

Additional file 1: Table S1. The information of Vicia faba L. germplasm used in this study.

Additional file 2: Table S2. The primer pairs were successfully designed by Primer3.

Additional file 3: Figure S1. Frequences of different SSR repeat motif types in mononuceotide.

Additional file 4: Figure S2. Frequences of different SSR repeat motif types in dinuceotide.

Additional file 5: Frequences of different SSR repeat motif types in trinuceotide.

Additional file 6: Figure S4. Frequences of different SSR repeat motif types in tetranuceotide.

Additional file 7: Figure S5. Figure S3. Frequences of different SSR repeat motif types in pentanuceotide.

Additional file 8: Figure S6. Frequences of different SSR repeat motif types in heaxanuceotide.

\section{Abbreviations}

SSR: Simple sequence repeat; QTL: Quantative Trait Locus; MAS: Markerassisted selection; NGS: Next generation sequencing; EST: Express sequence tag; NCBI: National Center for Biotechnology Information;

CTAB: Cetyltrimethylammonium bromid; MISA: Microsatellite identification; $\mathrm{Na}$ : Number of alleles; He: Expected heterozygosities; Ho: Observed heterozygosities.

\section{Competing interests}

The authors declare that they have no competing interests. 


\section{Authors' contributions}

TY performed bioinformatic analysis, primer design and drafted the manuscript. SYB created the SSR sequences rich DNA library, and participated in 454 sequencing. RF assisted in designing experiment and preparing the manuscript. TJJ tested SSR markers. JPG and YHH prepared al the seeds of $V$. faba. XLS and JYJ took charge of quality inspection of the DNA library. JJH and XYZ participated in conceiving the study and the manuscript drafting. XXZ designed and coordinated the study, and assisted in preparing the manuscript. All authors read and approved the final manuscript.

\section{Acknowledgements}

This work was supported by the National Natural Science Foundation of China (no. 31101198), the China Agriculture Research System (CARS-09) from the Ministry of Agriculture of China and Social Development of Science and Technology Plan (no. 2010BB007) from Yunnan Government. We are grateful to Dr. Dahai Wang and Liping Sun (Beijing Autolab Biotechnology Co., Ltd) for their special contribution to this work

\section{Author details}

'Institute of Crop Science, The National Key Facility for Crop Gene Resources and Genetic Improvement, Chinese Academy of Agricultural Sciences, Beijing 100081, China. ${ }^{2}$ Institute of Grain Crops, Yunnan Academy of Agricultural Sciences, Kunming 650205, China. ${ }^{3}$ Department of Agriculture and Food Systems, Melbourne School of Land and Environment, The University of Melbourne, Melbourne, Victoria 3010, Australia. ${ }^{4}$ Qingdao Academy of Agricultural Sciences, Qingdao 266100, China.

Received: 19 July 2012 Accepted: 4 November 2012

Published: 8 November 2012

\section{References}

1. Rispail N, Kal P, Kiss GB, Ellis THN, Gallardo K, Thompson RD, Prats E, Larrainzar E, Ladrera R, Gonzalez EM, Arreselgor C, Ferguson BJ, Gresshoff PM, Rubiales D: Model legumes contribute to faba bean breeding. Field Crop Res 2010, 115:253-269.

2. Duc G, Marget P, Esnault R, Le Guen J, Bastianelli D: Genetic variability for feeding value of faba bean seeds (Vicia faba): Comparative chemical composition of isogenics involving zero-tannin and zerovicine genes. J Agric Sci 1999, 133:185-196.

3. FAOSTAT: 2010. http://faostat.fao.org/site/291/default.aspx

4. Johnston JS, Bennett MD, Rayburn AL, Galbraith DW, Price HJ: Reference standards for determination of DNA content of plant nuclei. Am J Bot 1999, 86:609.

5. Morozova O, Marra MA: Applications of next-generation sequencing technologies in functional genomics. Genomics 2008, 92:255-264.

6. Mardis ER: The impact of next-generation sequencing technology on genetics. Trends Genet 2008, 24:133-141.

7. Lam HM, Xu X, Liu X, Chen W, Yang G, Wong FL, Li MW, He W, Qin N, Wang B, Li J, Jian M, Wang J, Shao G, Wang J, Sun SSM, Zhang G: Resequencing of 31 wild and cultivated soybean genomes identifies patterns of genetic diversity and selection. Nat Genet 2010, 42:1053-1059.

8. Huang X, Wei X, Sang T, Zhao Q, Feng Q, Zhao Y, Li C, Zhu C, Lu T, Zhang Z, Li M, Fan D, Guo Y, Wang A, Wang L, Deng L, Li W, Lu Y, Weng Q, Liu K, Huang T, Zhou T, Jing Y, Li W, Lin Z, Buckler ES, Qian Q, Zhang QF, Li J, Han B: Genomewide association studies of 14 agronomic traits in rice landraces. Nat Genet 2010, 42:961-967.

9. Tautz D, Renz M: Simple sequences are ubiquitous repetitive components of eukaryotic genomes. Nucleic Acids Res 1984, 12:4127-4138.

10. Lagercrantz $U$, Ellegren $H$, Andersson $L$ : The abundance of various polymorphic microsatellite motifs differs between plants and vertebrates. Nucleic Acids Res 1993, 21:1111-1115.

11. Rafalski JA, Tingey SV: Genetic diagnostics in plant breeding: RAPDs, microsatellites and machines. Trends Genet 1993, 9:275-280.

12. Požárková D, Kobližková A, Román B, Torres AM, Lucretti S, Lysák M, Doležel J, Macas J: Development and characterization of microsatellite markers from chromosome 1-specific DNA libraries of Vicia faba. Biologia Plantarum 2002, 45:337-345.

13. Zeid M, Mitchell S, Link W, Carter M, Nawar A, Fulton T, Kresovich S: Simple sequence repeats (SSRs) in faba bean: new loci from Orobancheresistant cultivar 'Giza 402'. Plant Breeding 2009, 128:149-155.
14. Gong Y-M, Xu S-C, Mao W-H, Hu Q-Z, Zhang G-W, Ding J, Li Z-Y: Generation and characterization of 11 novel EST derived microsatellites from Vicia faba (Fabaceae). Am J Bot 2010, 97:e69-e71.

15. Ma Y, Yang T, Guan J, Wang S, Wang H, Sun X, Zong X: Development and characterization of 21 EST-derived microsatellite markers in Vicia faba (fava bean). Am J Bot 2011, 98:e22-e24.

16. Zhang WK, Wang YJ, Luo GZ, Zhang JS, He CY, Wu XL, Gai JY, Chen SY: QTL mapping of ten agronomic traits on the soybean (Glycine max L. Merr.) genetic map and their association with EST markers. Theor Appl Genet 2004, 108:1131-1139.

17. Matthews BF, Devine TE, Weisemann JM, Beard HS, Lewers KS, MacDonald MH, Park Y-B, Maiti R, Lin J-J, Kuo J, Pedroni MJ, Cregan PB, Saunders JA: Incorporation of sequenced CDNA and genomic markers into the soybean genetic map. Crop Science 2001, 41:516-521.

18. Hisano H, Sato S, Isobe $S$, Sasamoto S, Wada T, Matsuno A, Fujishiro T, Yamada M, Nakayama S, Nakamura Y, Watanabe S, Harada K, Tabata S: Characterization of the soybean genome using EST-derived microsatellite markers. DNA Res 2007, 14:271-281.

19. Dellaporta S, Wood J, Hicks J: A plant DNA minipreparation: Version II. Plant Molecular Biology Reporter 1983, 1:19-21.

20. Doyle JJ, Doyle JL: A rapid total DNA preparation procedure for fresh plant tissue. Focus 1990, 12:13-15.

21. Kandpal RP, Kandpal G, Weissman SM: Construction of libraries enriched for sequence repeats and jumping clones, and hybridization selection for region-specific markers. Proc Natl Acad Sci USA 1994, 91:88-92.

22. Armour JA, Neumann R, Gobert S, Jeffreys AJ: Isolation of human simple repeat loci by hybridization selection. Hum Mol Genet 1994 3:599-565

23. Glenn TC, Schable NA: Isolating microsatellite DNA loci. Methods Enzymol 2005, 395:202-222.

24. Rice P, Longden I, Bleasby A: EMBOSS: the European molecular biology open software suite. Trends Genet 2000, 16:276-277.

25. Thiel, Michalek, Varshney, Graner: Exploiting EST databases for the development and characterization of gene-derived SSR-markers in barley (Hordeum vulgare L.). Theor Appl Genet 2003, 106:411-422.

26. Yeh FC, Boyle TJB: Population genetic analysis of co-dominant and dominant markers and quantitative traits. Belgian Journal of Botany 1997, 129:157.

27. Nei M: Estimation of average heterozygosity and genetic distance from a small number of individuals. Genetics 1978, 89:583-590.

28. Tamura K, Dudley J, Nei M, Kumar S: MEGA4: molecular evolutionary genetics analysis (MEGA) software version 4.0. Mol Biol Evol 2007, 24:1596-1599.

29. Zong X, Liu X, Guan J, Wang S, Liu Q, Paull JG, Redden R: Molecular variation among Chinese and global winter faba bean germplasm. Theor Appl Genet 2009, 118:971-978.

30. Wang HF, Zong XX, Guan JJ, Yang T, Sun XL, Ma Y, Redden R: Genetic diversity and relationship of global faba bean (Vicia faba L.) germplasm revealed by ISSR markers. Theor Appl Genet 2012, 124:789-797.

31. Hearne CM, Ghosh S, Todd JA: Microsatellites for linkage analysis of genetic traits. Trends Genet 1992, 8:288-294.

32. Diwan N, Cregan PB: Automated sizing of fluorescent-labeled simple sequence repeat (SSR) markers to assay genetic variation in soybean. Theor Appl Genet 1997, 95:723-733.

33. Edwards A, Civitello A, Hammond HA, Caskey CT: DNA typing and genetic mapping with trimeric and tetrameric tandem repeats. Am J Hum Genet 1991, 49:746-756

34. Gastier JM, Pulido JC, Sunden S, Brody T, Buetow KH, Murray JC, Weber JL, Hudson TJ, Sheffield VC, Duyk GM: Survey of trinucleotide repeats in the human genome: assessment of their utility as genetic markers. Hum Mol Genet 1995, 4:1829-1836

35. Sheffield VC, Weber JL, Buetow KH, Murray JC, Even DA, Wiles K, Gastier JM, Pulido JC, Yandava C, Sunden SL, Mattes G, Businga T, McClain A, Beck J, Scherpler T, Gilliam J, Zhong J, Duyk GM: A collection of tri- and tetranucleotide repeat markers used to generate high quality, high resolution human genome-wide linkage maps. Hum Mol Genet 1995 4:1837-1844.

36. Sonah H, Deshmukh RK, Sharma A, Singh VP, Gupta DK, Gacche RN, Rana JC, Singh NK, Sharma TR: Genome-wide distribution and organization of microsatellites in plants: an insight into marker development in Brachypodium. PLoS One 2011, 6:e21298. 
37. Pfeiffer $\mathrm{A}$, Olivieri $\mathrm{AM}$, Morgante M: Identification and characterization of microsatellites in Norway spruce (Picea abies K.). Genome 1997, 40:411-419.

38. Smith D, Devey ME: Occurrence and inheritance of microsatellites in Pinus radiata. Genome 1994, 37:977-983.

39. Roder MS, Plaschke J, Konig SU, Borner A, Sorrells ME, Tanksley SD, Ganal MW: Abundance, variability and chromosomal location of microsatellites in wheat. Mol Gen Genet 1995, 246:327-333.

40. Kaur S, Pembleton LW, Cogan NO, Savin KW, Leonforte T, Paull J, Materne M, Forster JW: Transcriptome sequencing of field pea and faba bean for discovery and validation of SSR genetic markers. BMC Genomics 2012, 13:104.

41. Zong X, Redden RJ, Liu Q, Wang S, Guan J, Liu J, Xu Y, Liu X, Gu J, Yan L, Ades $P$, Ford R: Analysis of a diverse global Pisum sp. collection and comparison to a Chinese local $P$. sativum collection with microsatellite markers. Theor Appl Genet 2009, 118:193-204.

doi:10.1186/1471-2164-13-602

Cite this article as: Yang et al:: High-throughput novel microsatellite marker of faba bean via next generation sequencing. BMC Genomics 2012 13:602.

\section{Submit your next manuscript to BioMed Central and take full advantage of:}

- Convenient online submission

- Thorough peer review

- No space constraints or color figure charges

- Immediate publication on acceptance

- Inclusion in PubMed, CAS, Scopus and Google Scholar

- Research which is freely available for redistribution 